\title{
Two-Dimensional Quasicrystal with Eightfold Rotational Symmetry
}

\author{
N. Wang, H. Chen, and K. H. Kuo \\ Beijing Laboratory of Electron Microscopy, Chinese Academy of Sciences, Beijing, China, and \\ Laboratory of Atomic Imaging of Solids, Institute of Metal Research, Chinese Academy of Science, Shenyang, Liaoning, China \\ (Received 12 May 1987)
}

\begin{abstract}
A 2D quasicrystal with an eightfold rotational axis has been found in rapidly solidified V-Ni-Si and $\mathrm{Cr}-\mathrm{Ni}-\mathrm{Si}$ alloys by means of transmission electron microscopy. The electron-diffraction pattern taken along this axis shows no periodicity but a clear eightfold orientation symmetry. The corresponding high-resolution electron-microscopic image agrees well with the $2 \mathrm{D}$ eightfold quasilattice consisting of squares and $45^{\circ}$ rhombi.
\end{abstract}

PACS numbers: $61.55 . \mathrm{Hg}, 61.50 . \mathrm{Em}$

Since the sensational discovery of the icosahedral quasicrystal first in $\mathrm{Al}-M(M=\mathrm{Cr}, \mathrm{Mn}, \mathrm{Fe})$ alloys ${ }^{1}$ and somewhat later in $\mathrm{Ni}-\mathrm{Ti}-\mathrm{V}^{2}$ and $\mathrm{Pd}-\mathrm{U}-\mathrm{Si}^{3}$ alloys, quasicrystals with tenfold ${ }^{4-6}$ and twelvefold ${ }^{7}$ rotational symmetries have also been reported. These symmetries are not compatible with the translational symmetry of the Bravais lattices, and the latter is considered to be an essential element of the classical crystallography. Though Pauling ${ }^{8}$ and others still advocate the multipletwin explanation of the icosahedral symmetry, the large amount of accumulated experimental facts and theoretical analyses ${ }^{9}$ seems to have already proved the very existence of quasicrystals with noncrystallographic symmetry. We report here the discovery of a new type of twodimensional (2D) quasicrystal with eightfold rotational symmetry in rapidly solidified $\mathrm{Cr}_{5} \mathrm{Ni}_{3} \mathrm{Si}_{2}$ and $\mathrm{V}_{15} \mathrm{Ni}_{10} \mathrm{Si}$ alloys and propose to call it the octagonal phase.

The alloy films used in this investigation were prepared first by melting pure metals and silicon in an $\mathrm{Ar}$ arc furnace followed by remelting and rapid solidification (about $10^{6} \mathrm{deg} / \mathrm{sec}$ ) by the piston-and-anvil method. The foils suitable for transmission electron microscopy were prepared from these films by electrolytical thinning in $5 \% \mathrm{HClO}_{4}$ in ethanol at $-30^{\circ} \mathrm{C}$ followed by a brief ion thinning. They were examined in a Philips EM420 electron microscope equipped with a large-angle double tilting stage $\left( \pm 45^{\circ}, \pm 60^{\circ}\right)$ so that electron-diffraction patterns (EDP) can sometimes be obtained from a single grain with the eightfold axis either parallel or perpendicular to the electron beam. The high-resolution electron-
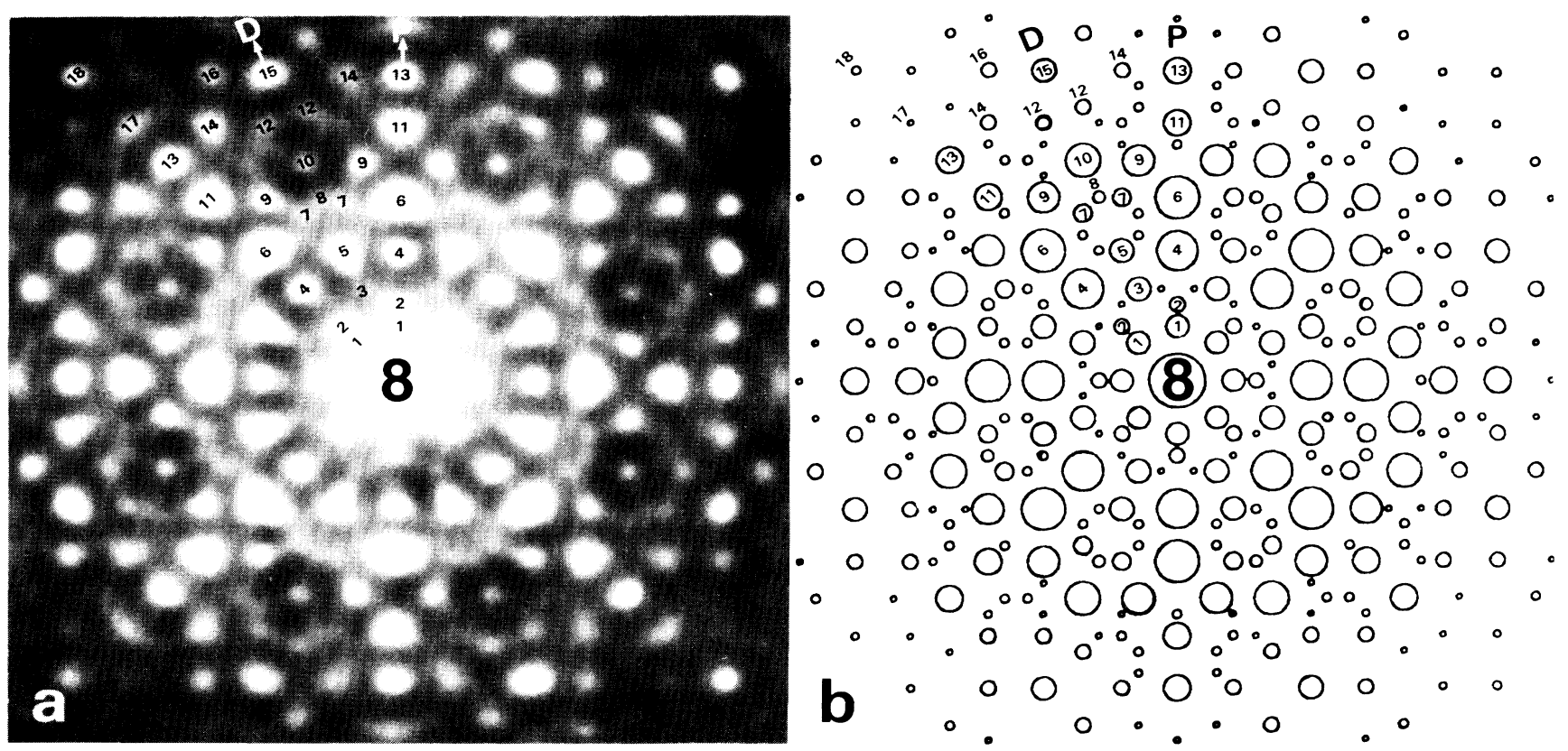

FIG. 1. (a) Selected-area electron diffraction-pattern from the Cr-Ni-Si octagonal phase with its eightfold axis parallel to the electron beam. (b) Simulated diffraction pattern of a 2D eightfold quasilattice [Fig. 3(a)]. 
microscopic (HREM) images were taken in a JEM200CX electron microscope equipped with a top entry stage working at $200 \mathrm{kV}$ with an interpretable resolution of $0.25 \mathrm{~nm}$.

It was known from transmission-electron-microscopy studies of icosahedral and decagonal quasicrystals that usually they have a mottled appearance, and this was also true in the present case. Figure $1(a)$ is the EDP from such a mottled region and the eightfold distribution of spots is quite obvious. The octets of spots (sometimes double in number) in a $2 \pi / 8$ sector are marked with numerals in order of increasing distance from the center. These spots do not lie on a cross grid as in an EDP of a crystal. In other words, no simple periodicity can be derived from this EDP which is a typical feature of a quasicrystal. The ratio of spacings between neighboring spots in a row, such as spots $1,2,4,6,11$, and 13 in row $P$ or spots $3,5,8,10$, and 15 in row $D$, is either $\sqrt{2}$ or $1+\sqrt{2}$, both of which associated with the angle of $2 \pi / 8$. Groups of spots very often have the configuration of an octagon, such as spots $7,9,12$, etc., around the spot 10 , or squares of different sizes. All these are in harmony with the eightfold symmetry and such an EDP has not yet been reported, though the possibility of $2 \mathrm{D}$ eightfold symmetry has recently been discussed by several groups of investigators working with quasicrystals. ${ }^{10-15}$
Figure 1(a) was taken with the eightfold axis parallel to the electron beam. By tilting the foil through $90^{\circ}$ around the axis of spots 1 and 13 (row $P$ ) or 3 and 15 (row $D$ ), respectively, the eightfold axis was brought successively down to a direction perpendicular to the electron beam (Fig. 2). As in the case of the 2D decagonal quasicrystal, all spots now lie on equally spaced, straight lines, though alternate rows are extinctions in the pattern $P$. This means a 2D quasicrystal with a one-dimensional periodicity along the eightfold axis, and the period determined from the pattern $D$ is about 0.63 $\mathrm{nm}$, which will be further discussed later. This $+22.5^{\circ}$ sector is a repeating unit and the $-22.5^{\circ}$ sector can be obtained from it by a reflection across the line $8 P$. Combining Figs. 1(a) and 2, it seems that what is under examination may be an eightfold 2D quasicrystal.

The angles associated with eightfold symmetry are integral multiples of $2 \pi / 8$ which can be found in squares and $45^{\circ}$ rhombi. The quasiperiodic combination of these two geometrical shapes was discussed first by Ammann ${ }^{16}$ in connection with tiling a plane nonperiodically, and somewhat later by Beenker ${ }^{17}$ in an algebraic analysis. Figure 3(a) was drawn by a computer program written by Ms. Z. M. Wang based on the theory of Levine and Steinhardt. ${ }^{18}$ Local eightfold symmetry can be found in many places with eight differently oriented $45^{\circ}$ rhombi

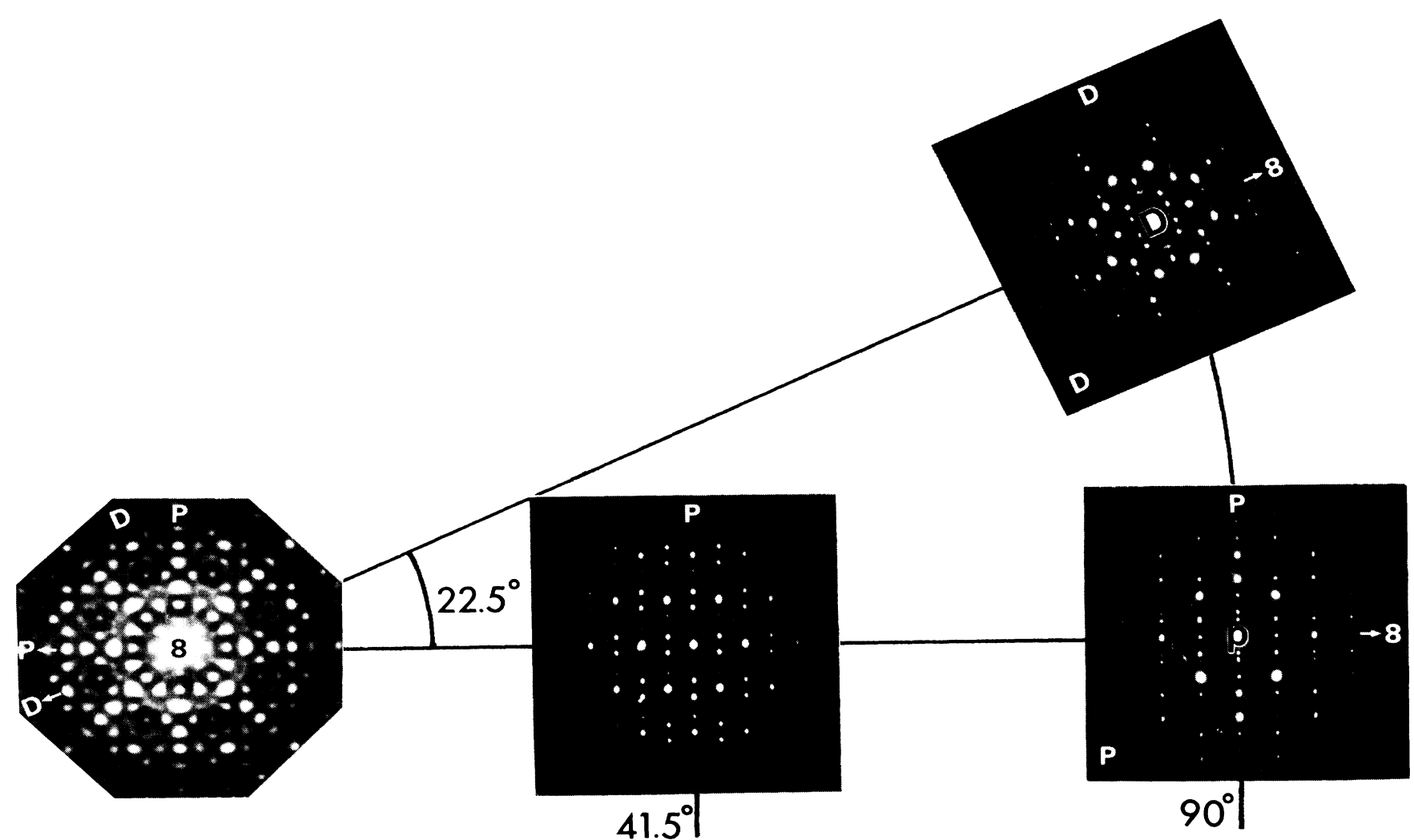

FIG. 2. Selected-area electron-diffraction patterns taken from a single grain of the $\mathrm{Cr}$-Ni-Si octagonal phase. 


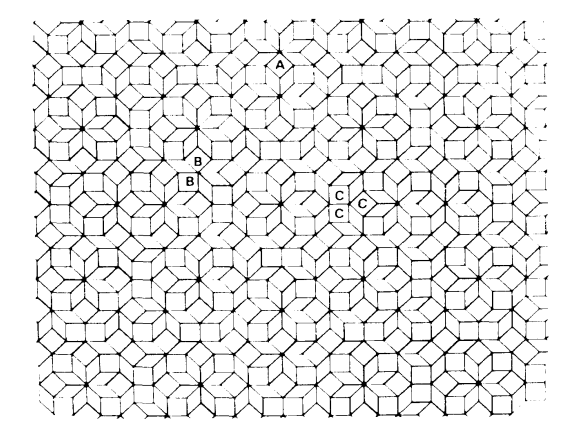

(a)

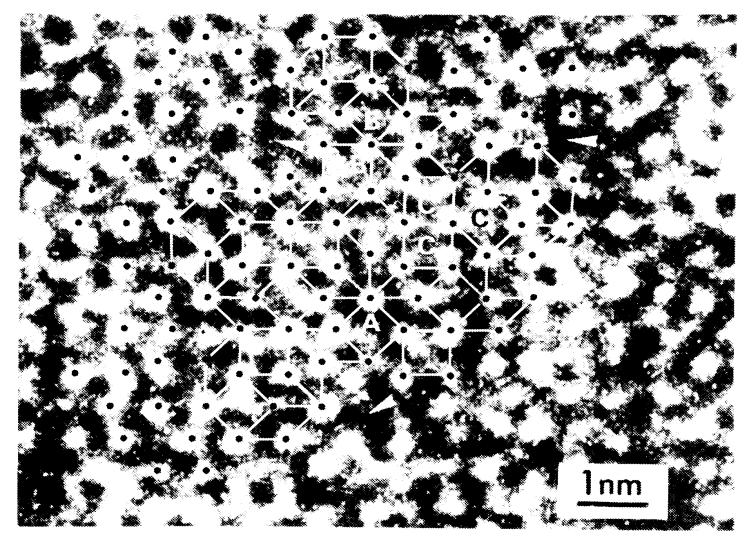

(b)

FIG. 3. (a) $2 \mathrm{D}$ octagonal quasilattice consisting of squares and $45^{\circ}$ rhombi. $A, B$, and $C$ mark the junctions of one square and six rhombi, two squares and four rhombi, and three squares and two rhombi, respectively. (b) High-resolution electron-microscopic image of the $\mathrm{Cr}-\mathrm{Ni}-\mathrm{Si}$ octagonal phase. Squares and $45^{\circ}$ rhombi are outlined to show the presence of many octagons. Configurations similar to $A, B$, and $C$ in Fig. 3 (a) are also marked.

sharing a common vertex. On the other hand, the squares can only have two orientations at $45^{\circ}$ with each other and at most only two squares of the same orientation can occur together. The Fourier transform of Fig. 3(a) calculated according to Levine and Steinhardt ${ }^{18}$ is shown in Fig. 1(b) which matches Fig. 1(a) rather well. Watanabe, Ito, and Soma ${ }^{15}$ have calculated a similar Fourier transform, but only the inner 6 or 7 octets of spots were included. All peaks in Fig. 1(b) occur at exactly the same position as the spots in the eightfold EDP in Fig. 1(a). Figure 3(b) is the HREM image of this octagonal phase. With the exception of some defects marked with arrow heads, most bright dots are arranged on an eightfold pattern of squares and $45^{\circ}$ rhombi. Beenker ${ }^{17}$ has analyzed the possible junctions of these geometrical shapes, such as one square and six rhombi, two squares and four rhombi, and three squares and two rhombi $[A, B$, and $C$, respectively, in Fig. 3(a)]. All these configurations have been observed in Fig. 3(b). In

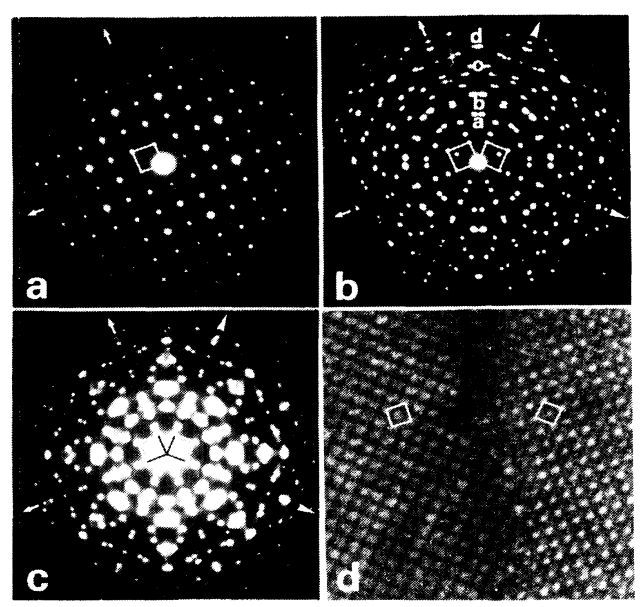

FIG. 4. (a) [001] EDP of a single variant (b), $45^{\circ}$ twins, and (c) microtwins of a domain size of about 10-20 nm of the $\beta$-Mn structure. (d) High-resolution electron-microscopic image of a pair of $45^{\circ}$ twins (the linear dimension of the square and rhombus is $0.63 \mathrm{~nm}$ ).

another HREM image, eight rhombi sharing a common vertex have also been observed. Furthermore, many octagons consisting of two squares and four rhombi, all differently oriented, are outlined in this image to show its octagonal character. The close similarity between the observed configuration of bright dots and the quasiperiodic eightfold pattern may possibly serve to show the existence of the octagonal quasicrystal in the rapidly solidified $\mathrm{Cr}-\mathrm{Ni}$-Si and $\mathrm{V}-\mathrm{Ni}-\mathrm{Si}$ alloys.

It should be pointed out that a kind of $\beta$ - Mn structure (space group $P 4_{1} 32, a=0.63 \mathrm{~nm}$ ) has been found coexisting with this octagonal phase in both $\mathrm{Cr}-\mathrm{Ni}-\mathrm{Si}$ and $\mathrm{V}$ $\mathrm{Ni}-\mathrm{Si}$ alloys after rapid solidification, and it occurs very often as $45^{\circ}$ rotational twins ssee the [001] EDP of a single variant in Fig. 4(a) and that of $45^{\circ}$ twins in Fig. 4(b) $\}$. The [001] EDP of the $45^{\circ}$ twins displays a pseudo-eightfold symmetry of diffraction spots and this becomes more obvious when the domain size decreases down to $10-20 \mathrm{~nm}$ [Fig. 4(c)]. The 210, 310, 420, and 520 spot pairs $[a, b, c$, and $d$ in Fig. 4(b)] of the twins merge together into strong broad discs, for instance, those in the vertical direction in Fig. 4(c), resembling the strong spots in row $P$ in Fig. 1 (a). Nevertheless, they do not occur at the exact same positions as spots $4,6,11$, and 13 in Fig. 1(a). Moreover, even at this small domain size the square array of $h k 0$ spots of these $45^{\circ}$ twins can still be recognized. Obviously, the spots in the $[100]^{*} /[110]^{*}$ direction (arrowed) are quite different from those in row $D$ in Fig. 1(a) and cannot be mistaken as those of the octagonal quasicrystal. Figure $4(d)$ is the [001] HREM image of a pair of $45^{\circ}$ microtwins of the $\beta$-Mn structure in which the square-planar lattices are outlined. When the $\beta$-Mn structure is projected onto (001), antisymmetrically superposed square units occur 
both at the corner and center of the square lattice and they are imaged as bright dots in Fig. 4(d). No matter how small the twins are, the square array of bright dots is the same and no $45^{\circ}$ rhombi can be detected in them. Maybe the $45^{\circ}$ rhombus unit can appear at a twin boundary, but the resolution of Fig. 4(d) is not good enough to show it. Anyway, the twin pattern in Fig. 4(d) is clearly distinct from Fig. 3(b), ruling out the possibility that the octagonal quasicrystal is in reality microtwins of the $\beta$-Mn structure.

Since the square unit in the octagonal phase in these alloys has a dimension of $0.63 \mathrm{~nm}$ and the periodicity along the eightfold axis is also $0.63 \mathrm{~nm}$, the cube in the octagonal phase is in fact a unit cell of the $\beta$-Mn structure. However, the spatial arrangement of these units is quite different, being periodic in the $\beta$ - $\mathrm{Mn}$ structure and quasiperiodic, together with $45^{\circ}$ rhombi, in the octagonal quasicrystal. Such a relation is analogous to that existing between icosahedral quasicrystals and FrankKasper phases ${ }^{19}$ or the complex structures of alloy phases in $\mathrm{Al}$-transition-metal systems. ${ }^{20}$

From the electron-diffraction pattern displaying eightfold symmetry and the corresponding high-resolution image resembling the eightfold quasiperiodic pattern, it is concluded that a $2 \mathrm{D}$ quasicrystal with eightfold rotational symmetry - the octagonal phase-has been observed in rapidly solidified $\mathrm{Cr}-\mathrm{Ni}-\mathrm{Si}$ and $\mathrm{V}-\mathrm{Ni}$-Si alloys. Similar findings have also occurred recently in rapidly solidified $\mathrm{Mn}-\mathrm{Fe}-\mathrm{Si}$ and $\mathrm{Mn}-\mathrm{Si}$ alloys in the Laboratory of Atomic Imaging of Solids, Institute of Metal Research, Chinese Academy of Sciencies, Shenyang.

The authors are grateful to Ms. Z. M. Wang of the Institute of Metal Research, Chinese Academy of Sciences, for the use of her computer programs to draw Figs. 1(b) and 3(a), and also to Mr. D. S. Zhou of the same institute for help in high-resolution electron microscopy.

${ }^{1}$ D. Shechtman, I. Blech, D. Gratias, and J. W. Cahn, Phys. Rev. Lett. 53, 1951 (1984).

${ }^{2}$ Z. Zhang, H. Q. Ye, and K. H. Kuo, Philos. Mag. 52, 141 (1985).

${ }^{3}$ S. J. Poon, A. J. Drehman, and K. R. Lawless, Phys. Rev. Lett. 55, 2324 (1985).

${ }^{4}$ L. Bendersky, Phys. Rev. Lett. 55, 1461 (1985).

${ }^{5}$ K. Chattopadhyay, S. Lele, S. Ranganathan, G. N. Subbanna, and N. Thangaraj, Curr. Sci. 54, 895 (1985).

${ }^{6}$ K. K. Fung, C. Y. Yang, Y. Q. Zhou, J. G. Zhao, W. S. Zhan, and B. G. Shen, Phys. Rev. Lett. 56, 2060 (1986).

${ }^{7}$ T. Ishimasa, H. U. Nissen, and Y. Fukano, Phys. Rev. Lett. 55, 511 (1985).

${ }^{8}$ L. Pauling, Phys. Rev. Lett. 58, 365 (1987).

${ }^{9}$ Proceedings of the International Workshop in Aperiodic Crystals, Les Houches, France 1986, edited by D. Gratias and L. Michel, J. Phys. (Paris), Colloq. 47, C3 (1986).

${ }^{10}$ D. M. Frenkel, C. L. Henley, and E. D. Siggia, Phys. Rev. B 34, 3649 (1986).

${ }^{11}$ D. Gratias and J. W. Cahn, Scr. Metall. 20, 1193 (1986).

${ }^{12}$ P. J. Steinhardt, Am. Sci. 74, 586 (1986).

${ }^{13}$ V. Sasisekharan, Pramana 26, 1283 (1986).

${ }^{14}$ T. Janssen, Acta. Crystallogr. Sect. A 42, 261 (1986).

${ }^{15}$ Y. Watanabe, M. Ito, and T. Soma, Acta Crystallogr. Sect. A 43, 133 (1987).

${ }^{16} \mathrm{R}$. Ammann, as quoted by R. Penrose, in Hermann Weyl 1885-1985, edited by K. Chandrasekharan (Springer-Verlag, Berlin, 1986), p. 23.

${ }^{17}$ F. P. M. Beenker, Technische Hogeschool Eindhoven Report No. 82-WSK-04, 1982 (unpublished).

${ }^{18}$ D. Levine and P. J. Steinhardt, Phys. Rev. Lett. 53, 2477 (1986).

${ }^{19}$ K. H. Kuo, J. Phys. (Paris), Colloq. 47, C3-425 (1986).

${ }^{20}$ C. L. Henley, Non-Cryst. Solid 75, 91 (1985). 

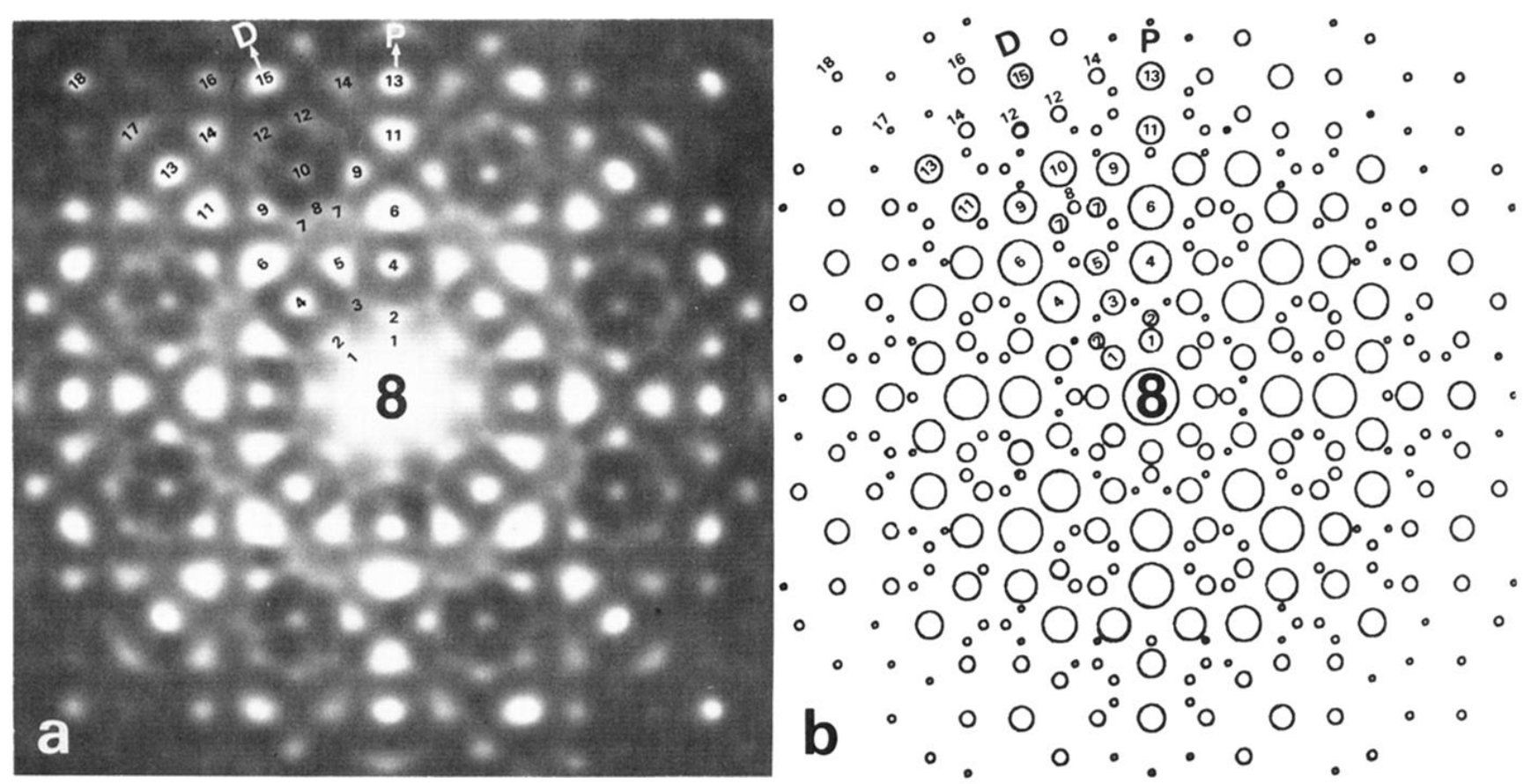

FIG. 1. (a) Selected-area electron diffraction-pattern from the Cr-Ni-Si octagonal phase with its eightfold axis parallel to the electron beam. (b) Simulated diffraction pattern of a 2D eightfold quasilattice [Fig. 3(a)]. 


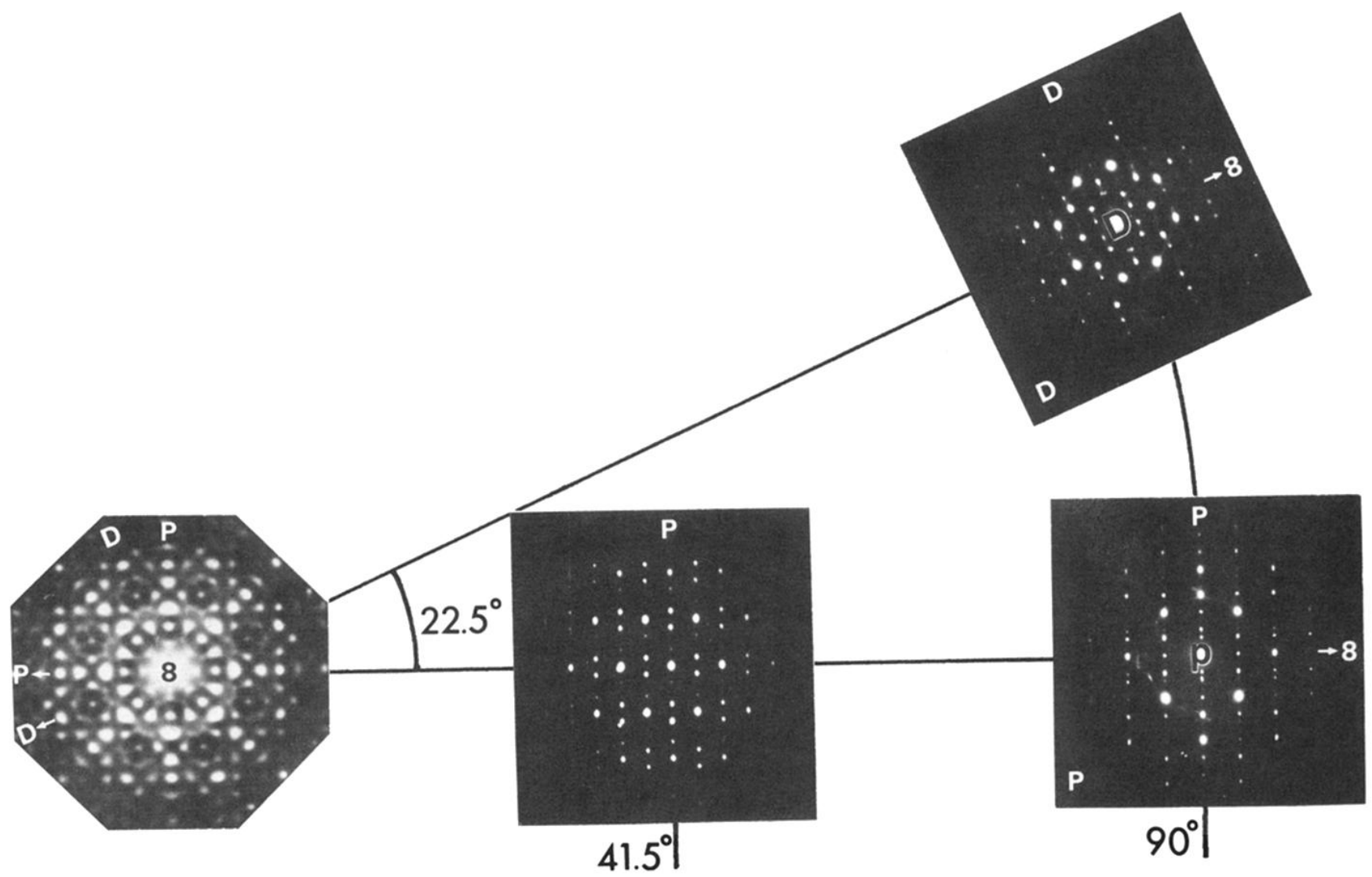

FIG. 2. Selected-area electron-diffraction patterns taken from a single grain of the $\mathrm{Cr}$-Ni-Si octagonal phase. 


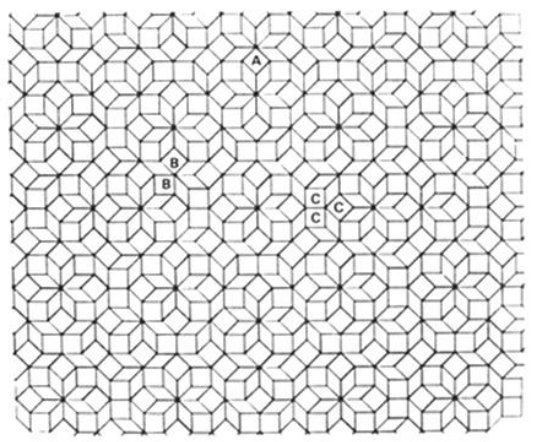

(a)

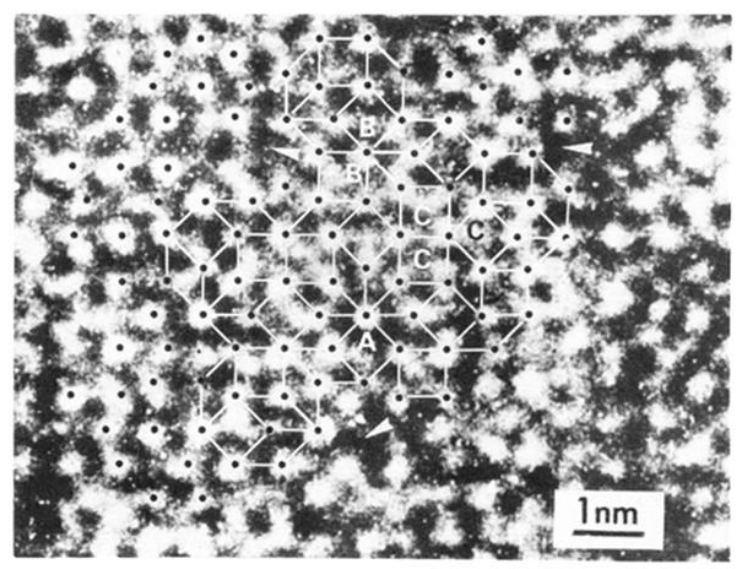

(b)

FIG. 3. (a) 2D octagonal quasilattice consisting of squares and $45^{\circ}$ rhombi. $A, B$, and $C$ mark the junctions of one square and six rhombi, two squares and four rhombi, and three squares and two rhombi, respectively. (b) High-resolution electron-microscopic image of the $\mathrm{Cr}-\mathrm{Ni}$-Si octagonal phase. Squares and $45^{\circ}$ rhombi are outlined to show the presence of many octagons. Configurations similar to $A, B$, and $C$ in Fig. 3(a) are also marked. 


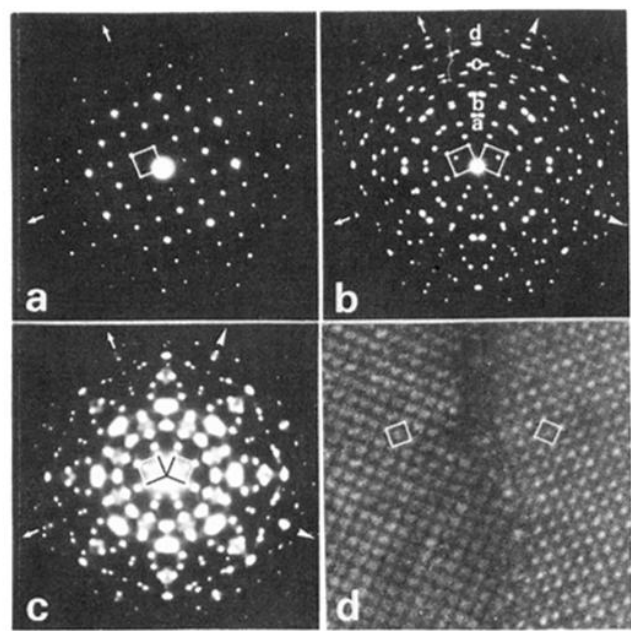

FIG. 4. (a) [001] EDP of a single variant (b), $45^{\circ}$ twins, and (c) microtwins of a domain size of about $10-20 \mathrm{~nm}$ of the $\beta$-Mn structure. (d) High-resolution electron-microscopic image of a pair of $45^{\circ}$ twins (the linear dimension of the square and rhombus is $0.63 \mathrm{~nm}$ ). 